\title{
An investigation of crystal structure, surface area and surface chemistry of strontium niobate and their influence on the photocatalytic performance.
}

\author{
Paraskevi Efstathiou ${ }^{1}$, Xiaoxiang $\mathrm{Xu}^{1}$, Hervé Ménard ${ }^{2}$ and John TS Irvine ${ }^{1 *}$ \\ 5 \\ 1. School of Chemistry, EaSTChem, University of St Andrews, St Andrews, Fife KY16 9ST, UK \\ 2. Sasol Technology (UK) Ltd., St. Andrews, KY16 9ST, Scotland, United Kingdom \\ E-mail: (jtsi@st-andrews.ac.uk) \\ Tel: +441334463817 \\ ${ }_{10}$ Fax: +44 1334463808 \\ n XXX, XXX) Xth XXXXXXXXX 20XX, Accepted Xth XXXXXXXXX 20XX \\ DOI: 10.1039/b000000X
}

$\mathrm{Sr}_{1-\mathrm{x}} \mathrm{NbO}_{3}$ is an unusual material that displays both metallic type conduction and photocatalytic activity, despite it being a NbIV oxide, it sustains photo-oxidation without degradation. ${ }^{1}$ The influence of 15 crystal structure, surface area and surface chemistry on the photocatalytic activity of strontium niobate has been investigated. The crystal structure of strontium niobate depends on the $\mathrm{Sr}$ content of the A site, with cubic symmetry for $\mathrm{Sr} \leq 0.92$ and orthorhombic symmetric for $0.92<\mathrm{Sr} \leq 0.97$. The change of crystal structure from cubic to orthorhombic symmetry seems to have a negative effect on the photocatalytic activity, as the $\mathrm{NbO}_{6}$ octahedra become distorted and unfavourable for d-orbital 20 overlapping. The photocatalytic activity increased significantly by enlarging the surface area through ball milling, nevertheless, a clear trend for surface area effect on activity is not obtained among samples with different Sr content. An enrichment of Sr on the surface of strontium niobate was observed by XPS, which seems to be a governing factor improving stability.

Keywords: Strontium niobate, photocatalyst, water splitting, visible light

\section{${ }_{25}$ Introduction}

Green energy is our civilization's hope in order to escape from the increasing and disastrous demand for fossil fuels. Photocatalysis is a method that converts light energy into chemical energy at an appropriate material (usually a 30 semiconductor) and through this process new fuels can be produced. Specifically in the case of water splitting under visible light, precious $\mathrm{H}_{2}$ and $\mathrm{O}_{2}$ are produced just by exploitation of the solar light. Unfortunately, very few materials have been found to be able to carry out the whole water splitting procedure and even 35 less can do that under visible, instead of UV, light ${ }^{2,3}$. Successful water splitting under visible light is often achieved by the production of a Z-scheme $\mathrm{Z}^{4-8}$, which is a system consisting of two photocatalysts, connected by a redox couple (e.g. $\mathrm{Fe}^{3+} / \mathrm{Fe}^{2+}$ ), one photocatalyst produces $\mathrm{H}_{2}$ and the other produces $\mathrm{O}_{2}$. However, 40 much study has been focused on materials' ability to complete the water splitting half reactions (either $\mathrm{H}_{2}$ or $\mathrm{O}_{2}$ production) using a sacrificial reagent. The fact that a material succeeds to photocatalytically produce either $\mathrm{H}_{2}$ or $\mathrm{O}_{2}$ with the help of a sacrificial reagent is a good indication of the materials potential 45 to photocatalytically fully split water. The most efficient and most widely studied material, although absorbing in the UV, with many applications is $\mathrm{TiO}_{2}{ }^{9-17}$ and the form which is found as being the most photocatalytically active is that of anatase as opposed to that of rutile ${ }^{9}$. However, given the importance of 50 exploiting as a larger amount of solar energy as possible the value of materials that can exhibit photocatalytic activity under visible light is profound.

Research to improve the photocatalytic process, focuses both on the enhancement of the efficiency of the photocatalytic 55 reactions, as well as on the design of the photocatalyst in order to be able to absorb and operate under solar or visible light. Some of the steps taken are: loading the photocatalyst with metal or oxide (e.g. small amounts of $\mathrm{Pt}$ or $\mathrm{NiO}$ ) that work as a co-catalysts, or introducing sacrificial reagents (e.g. oxalic acid) in order to 60 introduce active sites for $\mathrm{H}_{2}$ evolution, or facilitate hydrogen production by providing a more favourable oxidation path than water oxidation. These actions have proven to enhance the reactivity of the material ${ }^{15,18,19}$. Studies have also been carried out on the size of the semiconductor particles. It was found that 65 the smaller the particle size of the $\mathrm{TiO}_{2}$ catalyst, the more effective the photocatalytic reactions ${ }^{10,20}$. This is expected as a result of the increase of surface area, hence the increase of active 
sites on the surface of the photocatalyst. An effective way of achieving particle size decrease is ball milling ${ }^{20-22}$. Alongside surface area increase, more phenomena take place during the ball milling of the samples, some of which are potentially harmful to 5 the photocatalytic performance (e.g. decrease of crystallinity and creation of structural defects) ${ }^{20}$. Therefore, particular care is required during the process.

Many metal oxides have already been reported to be photocatalytically active under visible light ${ }^{22-25}$ using different 10 materials as sacrificial reagents. The majority of materials used for light harvesting applications are semiconductors, due to their ability when irradiated to produce electrons and holes, which separated by the band gap, constitute reactive reduction and oxidation sites respectively. However, strongly coloured metals 15 could substitute the use of semiconductors since the electron emissions between bands after irradiation could still achieve the necessary electron and hole production and the separation of which could be favoured by increased mobility. In this report we address such a strongly coloured metal oxide with metallic 20 behaviour, strontium niobate, as a possible photocatalyst that under visible light can produce $\mathrm{H}_{2}$ from the water splitting half reaction. In addition, we study the material's behaviour before and after a ball milling process intending to increase the material's surface area and correlate the different parameters that 25 determine the photocatalytic performance it exhibits.

In our previous work1, it has been shown that strontium niobate has successfully achieved the water splitting half-reaction of $\mathrm{H}_{2}$ production under visible light irradiation with oxalic acid as a sacrificial reagent, as well as the photoxidative degradation of 30 methylene blue and oxygen evolution from water with $\mathrm{Ag}^{+}$as sacrificial reagent. In addition, its conductivity was measured equal to $3 \times 10^{3} \mathrm{Scm}^{-1}$ for $\mathrm{x}=0.2$ and it was found to decrease slightly while the temperature increased, in agreement with what has already been reported in literature ${ }^{26-28}$. The temperature 35 coefficient of resistance was calculated to be $\alpha_{\mathrm{t}}=2.05 \mathrm{x} 10-4 \mathrm{~K}^{-1}$. Thus, the material is a red metallic oxide.

As a metal the excitation of electrons cannot be between the occupied valence (VB) and the almost empty conduction band (CB), as in the case of semiconductor, as the electronic structure 40 is quite different and the conduction band has a significant fraction of its states occupied. Instead excitation occurs between the highest fully occupied band $\left(\mathrm{B}_{1}\right)$ and the $\mathrm{CB}$ or the $\mathrm{CB}$ and the lowest unoccupied band $\left(\mathrm{B}_{-1}\right)$. In the case of strontium niobate the absorption energy gap, as taken by the UV-VIS 45 absorbance spectra, is $\sim 1.9 \mathrm{eV}$ (Fig.1).

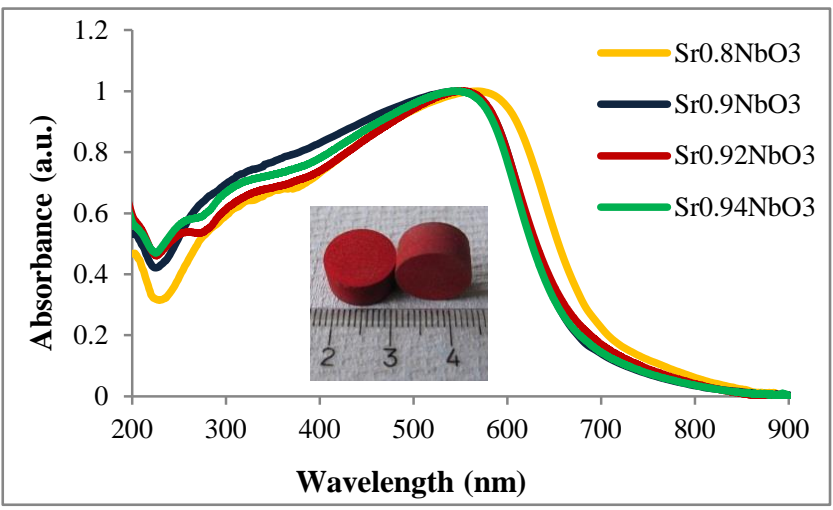

Fig.1 UV-VIS absorbance spectra (Converted from diffuse reflectance spectra) for $\mathrm{Sr}_{1-\mathrm{x}} \mathrm{NbO}_{3}(1-\mathrm{x}=0.8,0.9,0.92,0.94)$. Typical sintered pellets $\left(\mathrm{Sr}_{0.9} \mathrm{NbO}_{3}\right)$ are displayed in the inserted photo. Results for $(1-\mathrm{x}=0.8,0.9)$ 50 have been presented in our previous work ${ }^{1}$.

Strontium niobate is an oxide with perovskite structure. That means that it has the formula $\mathrm{ABO}_{3}$, where $\mathrm{A}$ and $\mathrm{B}$ are cations and it has an ideal cubic structure with $\mathrm{NbO}_{6}$ octahedra sharing 55 corners in all 3 dimensions. It can only be synthesised as A-site deficient $\left(\mathrm{Sr}_{1-\mathrm{x}} \mathrm{NbO}_{3}\right)$ for $0.3 \geq \mathrm{x} \geq 0.03$. Its colour changes from blue to red as the $\mathrm{Sr}$ content increases ${ }^{26,29}$. Above and below those limits the material gives complex XRD patterns revealing more than one phase. It has also been reported that its ideal cubic 60 structure is distorted to a lower symmetry structure, the orthorhombic one, for $\mathrm{x}<0.1$ and particularly for $\mathrm{x}=0.03^{26}$. Furthermore, the material is slightly oxygen deficient (small $\delta$ ) and at temperature higher than $300{ }^{\circ} \mathrm{C}$ it starts to get oxidized in air, as shown by thermogravimetric analysis (TGA) (Fig. 2). ${ }_{65}$ When fully oxidized, the material turns into a white, amorphous powder. This A-site deficiency and particularly the Sr content of the synthesized samples, seems to determine the material's characteristics correlating to the photocatalytic activity it exhibits. Specifically, the fact that while the $\mathrm{Sr}$ content increases the $\mathrm{Nb}$ 70 gets more reduced leads to changes not only in the crystal structure $^{26,29,31-33}$ (cubic $\rightarrow$ orthorhombic) but also to its electronic configuration, hence the absorption energy gap and the material's surface. Different phenomena take place during the ball milling of the samples too, with beneficial effect on the 75 photocatalytic performance (increase of surface area and oxidation of the surface).

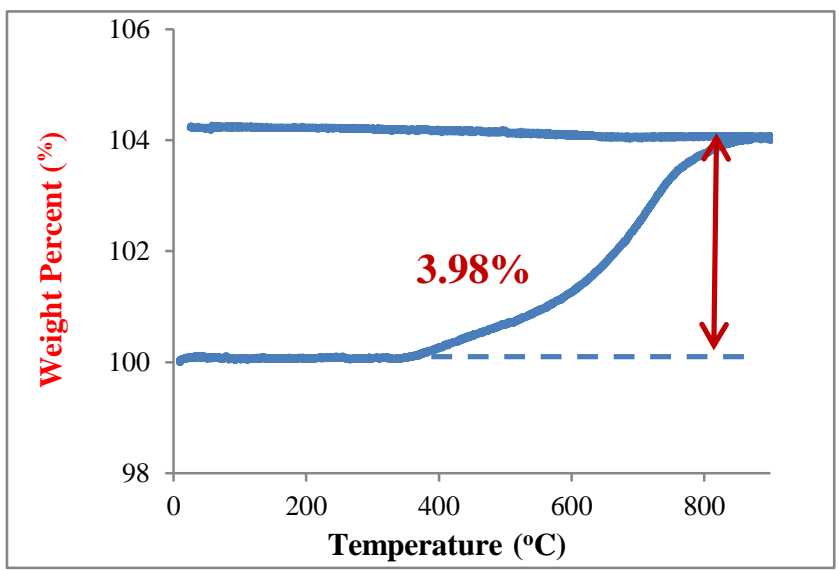

Fig. 2 TGA analysis of $\mathrm{Sr}_{0.9} \mathrm{NbO}_{3-\delta}$, heating up to $900{ }^{\circ} \mathrm{C}$ at $5^{\circ} \mathrm{min}^{-1}$ under air, holding for 5 minutes and cooling back to room temperature. The 80 material starts to be oxidized at $\sim 300{ }^{\circ} \mathrm{C}$. In this case $\delta$ is found to be 0.12 .

In this report, we have focused on the synthesis of the strontium niobate compound $\left(\mathrm{Sr}_{1-\mathrm{x}} \mathrm{NbO}_{3}\right)$ with different strontium 85 content, varying between $0.2 \geq x \geq 0.03$ and using it as a photocatalyst for the production of $\mathrm{H}_{2}$ from water splitting, using $0.025 \mathrm{M}$ oxalic acid as a sacrificial reagent. The compound's photocatalytic performance under visible light has been screened, both before and after ball milling, in order to determine the role 90 that the three important parameters play in the performance of the oxide: structure, surface area, surface morphology. 


\section{Experimental}

\section{Synthetic procedure}

Earlier syntheses of strontium niobates $\mathrm{Sr}_{1-\mathrm{x}} \mathrm{NbO}_{3}$ have encountered many difficulties, with pure samples of good 5 homogeneity being rather hard to obtain ${ }^{29,34}$. The challenges are the reaction conditions as well as the choice of precursors. Previous methods using $\mathrm{SrO}$ as a precursor in a vacuous quartz condition have many drawbacks, not only because $\mathrm{SrO}$ is sensitive towards $\mathrm{CO}_{2}$ and $\mathrm{H}_{2} \mathrm{O}$ but also, because it may react 10 with quartz to form stable silicates ${ }^{27,34}$. Later, replacing $\mathrm{SrO}$ with strontium niobate precursors such as $\mathrm{Sr}_{5} \mathrm{Nb}_{4} \mathrm{O}_{15}$ or $\mathrm{Sr}_{4} \mathrm{Nb}_{2} \mathrm{O}_{9}$ was very helpful but a long reaction time was still needed ${ }^{27}$. This was due to the low reaction temperatures that is limited by the softening temperature of quartz $\left(1200 \sim 1250{ }^{\circ} \mathrm{C}\right)^{29}$. Recent work

15 has used Niobium metal container (ampoules or tubes) under vacuum or in a flowing $\mathrm{Ar}$ conditions ${ }^{27,28,31}$. Here we use $\mathrm{Sr}_{4} \mathrm{Nb}_{2} \mathrm{O}_{9}$ as a precursor and the reaction is performed in flowing Ar conditions.

The precursor powder $\mathrm{Sr}_{4} \mathrm{Nb}_{2} \mathrm{O}_{9}$ was mixed in 20 stoichiometric amounts with $\mathrm{Nb}$ metal (Alfa Aesar, 99.8\%, -325 mesh) in order to produce samples with $\mathrm{Sr}$ contents $(\mathrm{x}=0.2-0.03)$ that cover most of the range, for which, the material can be synthesized $^{29}$. After the two reagents are mixed, they are ground for at least 40 minutes with a mortar and pestle until the mixture 25 is as homogeneous as possible. Then pellets of $13 \mathrm{~mm}$ are pressed under a pressure of $50 \mathrm{MPa}$ and they are calcined, wrapped in molybdenum foil, at $1400{ }^{\circ} \mathrm{C}$ under reducing atmospheres (Ar) for $20 \mathrm{~h}$. Pellets of bright red colour are obtained. The precursor powder $\mathrm{Sr}_{4} \mathrm{Nb}_{2} \mathrm{O}_{9}$ is synthesised by mixing $\mathrm{SrCO}_{3}$ (Aldrich, $\geq$ 30 99.9\%) and $\mathrm{Nb}_{2} \mathrm{O}_{5}$ (Aldrich, 99.9\%, -325 mesh) in stoichiometric ratio and grinding until a homogeneous mixture is attained. Calcination at $1200{ }^{\circ} \mathrm{C}$ for $24 \mathrm{~h}$ follows, then the mixture is ground again and then the calcination process is repeated, before the precursor powder is successfully synthesized.

35 Electrical conductivity was measured on sintered pellets using a standard 4-probe DC method at room temperature $\left(20{ }^{\circ} \mathrm{C}\right)$. The measurement was carried out on a home-made jig with 4 terminals ${ }^{35}$. Each terminal is connected to a Pt strip with 1 mm thickness. All Pt strips are paralleled with each other with a

40 separation of $3 \mathrm{~mm}$. The inner two $\mathrm{Pt}$ strips were used as potential probes and the outer two as current probes. A current of $100 \mathrm{~mA}$ (model: Keithley 220, Keithley Instruments Inc, USA) was applied subsequently in both directions and the resistance was measured as a gradient of potential vs. current. The electrical 45 conductivity was then calculated using the geometrical factor of the pellets.

\section{Characterization}

X-ray diffraction (XRD) analysis took place on PANalytical Empyrean Series 2 diffractometer system to ensure 50 the purity and crystallinity of the samples, with step of $0.0167^{\circ}$ and data collection time $85,090 \mathrm{~s}$ at every step. Thermo- gravimetric analysis (TGA) to calculate the actual oxygen content of the material was done by heating up the samples from room temperature $\left(25{ }^{\circ} \mathrm{C}\right)$ to $900{ }^{\circ} \mathrm{C}$ under air and then cooling back ${ }_{55}$ down to room temperature. The ultraviolet - visible absorbance (UV - VIS) spectra were collected on a JASCO-V550 spectrophotometer and the band gap energies (Eg) were calculated by the Kubelka - Munk transformation. X-ray photoelectron spectroscopy (XPS) was performed on a Kratos 60 AXIS Ultra DLD, with a monochromatic Al K $\alpha(1486.6 \mathrm{eV}) \mathrm{X}$ ray source and for peak calibration adventitious $\mathrm{C}$ was used at $285.0 \mathrm{eV}$. Then the binding energy of the peaks acquired were compared and found in accordance to the ones reported in literature $^{34}$. The microstructure of samples was inspected by 65 scanning electron microscope (SEM) on a JEOL 5600 SEM. Ball milling was used to decrease the particle size of the material and increasing surface area in a Fritsch Planetary Micro Mill Pulverisette 7 using zirconia balls as milling media and isopropanol as a dispersant. The attained surface area was 70 measured in a Micromeritics ASAP 2020 V3.00 H surface area analyzer, with nitrogen as an adsorptive gas and the BET equation (Brunauer, Emmett and Teller, 1938) was used for the calculation. The particle size distribution (PSD) was carried out in a Matersizer 2000 Hydro 2000 s Malvern Instruments analyser 75 and isopropanol with $2 \%$ triton was used as a dispersant.

\section{Photocatalytic testing}

The photocatalytic activity of the samples was evaluated by their hydrogen production under visible light irradiation after a period of $18-26 \mathrm{~h}$. The lamp used is a $250 \mathrm{~W}$ 80 iron-doped metal halide ultraviolet-visible lamp with an UV cutoff filter ( $\lambda \geq 420 \mathrm{~nm}$; Borosilicate Coated Glass HM07, UQG (optic), Cambridge UK). An amount of $0.2 \mathrm{~g}$ of photocatalyst was used for the non ball milled samples and $0.1 \mathrm{~g}$ for the ball milled ones and it was dispersed in a quantity of $200 \mathrm{~mL}$ of aqueous 85 solution, which contained $0.025 \mathrm{M}$ oxalic acid as a sacrificial reagent. The reactor used is a home - made metallic box with PTFE lining on the inside and a quartz glass lid that seals tightly on top, where the irradiation comes through to reach the photocatalyst. A water jacket, with constant flow, on the outside 90 of the box keeps the temperature constant and close to room temperature. The $\mathrm{H}_{2}$ produced was measured by an online gas chromatographer (Agilent 3000 Micro Gas Chromatograph).

\section{Results and discussion}

\section{Phase compositions and conductivity}

95 Perovskite strontium niobate can exhibit different crystal structures depending on the $\mathrm{Sr}$ content in the A sites. Cubic symmetry (space group $\mathrm{P} \mathrm{m}-3 \mathrm{~m}$ ) has been reported when the occupancy of $\mathrm{Sr}$ lies between $(1-\mathrm{x})=0.75$ and $0.9^{27,28}$; while distorted structures with a higher Sr occupancy have been found 100 at room temperatures with decreased symmetries 26,31 . 


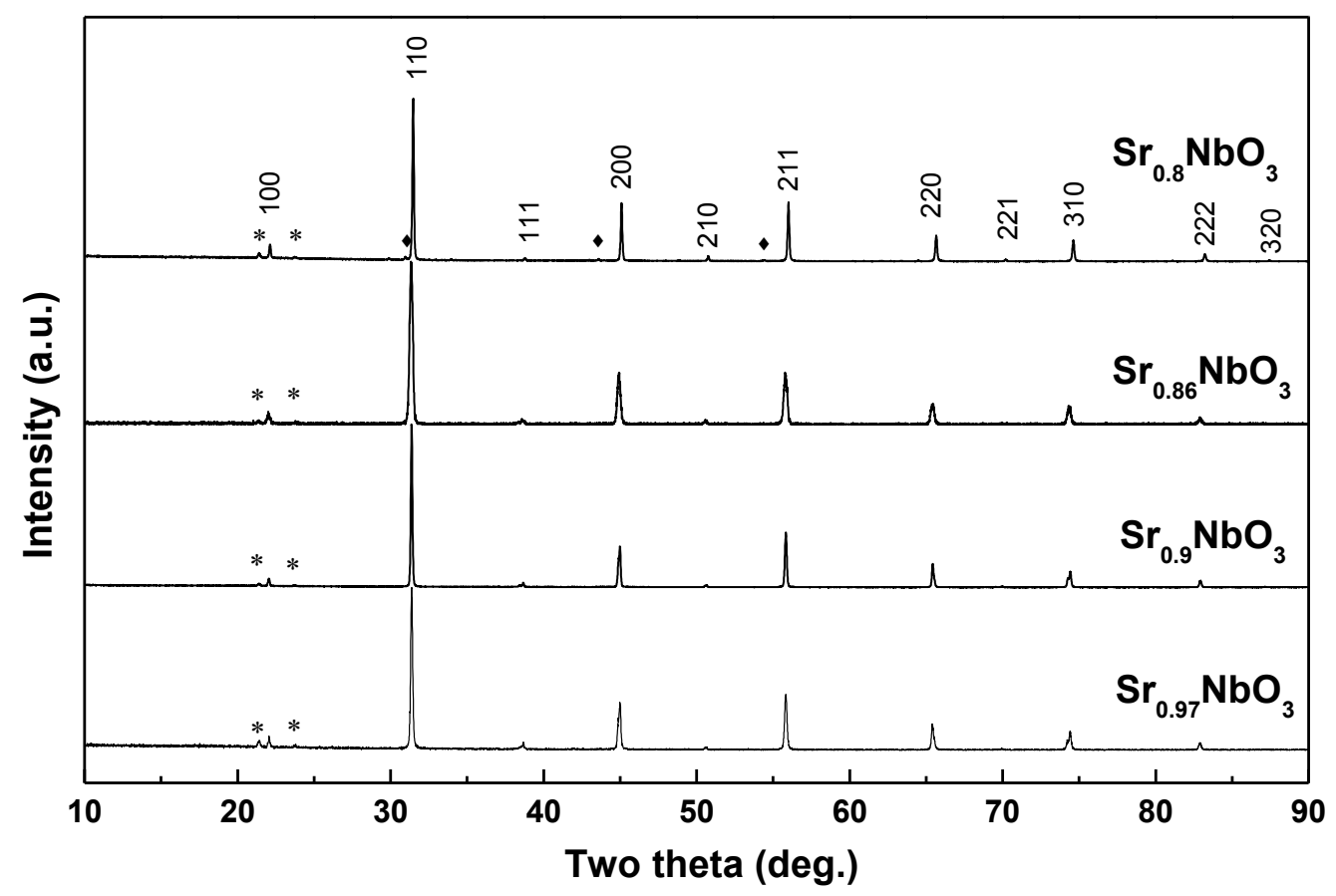

Fig. $3 \mathrm{X}$-ray powder diffraction patterns of $\mathrm{Sr}_{1-\mathrm{x}} \mathrm{NbO}_{3}(1-\mathrm{x}=0.97,0.9,0.86,0.8)$. Impurity peaks were labeled by diamond $(\diamond)$ and Vaseline peaks were marked by $(*)$. Results for $(1-\mathrm{x}=0.8,0.9)$ have been presented in our previous work ${ }^{1}$.

5 Table 1 Unit cell parameters, colour and structure of the as-prepared samples.

\begin{tabular}{ccccc} 
sample & $\mathrm{a}(\AA)$ & $\mathrm{V}\left(\AA^{3}\right)$ & Colour & Structure \\
$\mathrm{Sr}_{0.8} \mathrm{NbO}_{3}$ & $4.0194(6)$ & $64.94(3)$ & purple & Cubic \\
$\mathrm{Sr}_{0.86} \mathrm{NbO}_{3}$ & $4.0308(2)$ & $65.50(6)$ & red & Cubic \\
$\mathrm{Sr}_{0.9} \mathrm{NbO}_{3}$ & $4.0335(1)$ & $65.63(5)$ & red & Cubic \\
& $\mathrm{a}=5.7014(3)$ & & & \\
$\mathrm{Sr}_{0.97} \mathrm{NbO}_{3}$ & $\mathrm{~b}=5.6942(3)$ & $262.28(2)$ & red & Orthorhombic \\
& $\mathrm{c}=8.0786(3)$ & & & \\
\hline
\end{tabular}

More specifically, the material exhibits a cubic phase until around $x=0.08$ and from that point on, the $\mathrm{NbO}_{6}$ octahedra reorientate and the structure adopts the less symmetric form of 10 orthorhombic phase $\mathrm{e}^{26,29,31-33}$. In our synthesis, a series of samples $\left(\mathrm{Sr}_{1-\mathrm{x}} \mathrm{NbO}_{3-\delta}\right)$ were prepared with $\mathrm{Sr}$ content among 0.8 and 0.97 . XRD patterns of prepared samples can be indexed with cubic or orthorhombic symmetry (Fig. 3), consistent with previous results. Some impurity peaks were occasionally present in the patterns 15 but they were quite small. The refined unit cell parameters are listed in Table 1. A gradual increase in the unit cell parameters from $\mathrm{Sr}_{0.8} \mathrm{NbO}_{3}$ to $\mathrm{Sr}_{0.97} \mathrm{NbO}_{3}$ was observed and can be attributed to the decrease of $\mathrm{A}$ site vacancies as well as the reduction of $\mathrm{Nb}^{5+}$ (Ionic radius $0.64 \AA$ at 6 coordination) to $\mathrm{Nb}^{4+}$ (Ionic radius ${ }_{20} 0.69 \AA$ at 6 coordination) within the structure ${ }^{41}$.

The measured DC electrical conductivity at room temperatures for the samples was presented in our previous work ${ }^{1}$. Although the electrical conductivity is still lower than that of typical metals such as silver $\left(6.3 \times 10^{5} \mathrm{~S} / \mathrm{cm}\right)$ or copper $(5.96 \times$ ${ }_{25} 10^{5} \mathrm{~S} / \mathrm{cm}$ ), it is in the same order of magnitude as graphite or some metal alloys like Nichrome $\left(\sim 10^{3} \mathrm{~S} / \mathrm{cm}\right){ }^{37-39}$. Our previous 
measurement and other reports confirmed that the conductivity has a metallic behaviour, i.e. decreasing as the temperature increases. Therefore, they are essentially metallic conductors. However, the highest conductivity was observed for sample ${ }_{5} \mathrm{Sr}_{0.8} \mathrm{NbO}_{3}$ where $\mathrm{Nb}$ was least reduced (average valence +4.4) among all measured samples. Prior reports show similar magnitudes ${ }^{27,28}$, although values do vary probably due to differing microstructures of the sintered pellets.

From SEM images (Fig. 4), it can be seen that the 10 freshly sintered pellets all present a porous surface with particle sizes as large as several microns. The measured density for all pellets only reaches around $56 \%$ of the theoretical density.

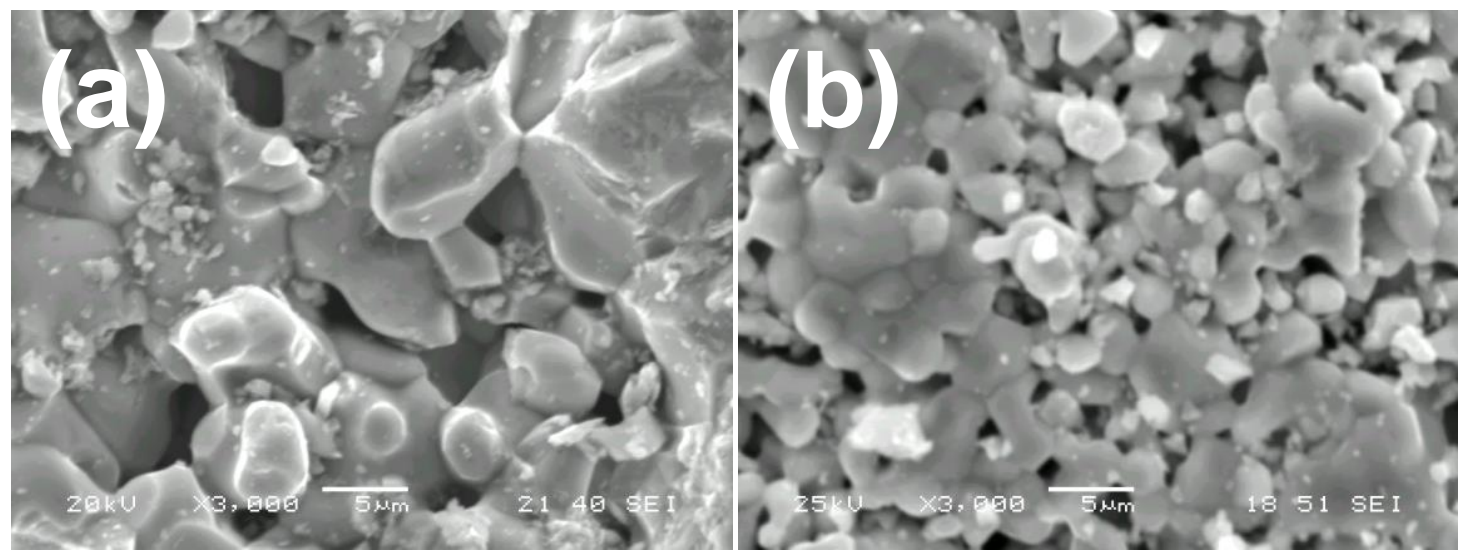

Fig. 4 SEM images from the surface of the sintered pellets, (a) $\mathrm{Sr}_{0.9} \mathrm{NbO}_{3}$ and (b) $\mathrm{Sr}_{0.8} \mathrm{NbO}_{3}$ showing particle size of around $10 \mu$.

\section{UV-VIS Diffuse reflectance spectra}

The optical absorption spectra of the sample powders are displayed in Fig. 1. The spectra were converted from diffuse reflectance spectra and Mubelka-Munk transformation was ${ }_{20}$ performed to help with the determination of the band gap. The red or purple colour of $\mathrm{Sr}_{1-\mathrm{x}} \mathrm{NbO}_{3}$ is justified by the absorption tail extending to nearly $700 \mathrm{~nm}$. The corresponding energy gap calculated by extrapolating the onset of Mubelka-Munk curves to the energy axis is around $1.9 \mathrm{eV}$. Theoretical calculations based 25 on the wavelength-intensity distribution of sunlight suggest an optimal band gap of $1.5 \pm 0.5 \mathrm{eV}$ for the maximum conversion efficiency ${ }^{44}$, therefore $\mathrm{Sr}_{1-\mathrm{x}} \mathrm{NbO}_{3}$ can be a promising candidate for solar energy utilisation.

\section{${ }_{30}$ Photocatalytic performance}

The photocatalytic activity of $\mathrm{Sr}_{1-\mathrm{x}} \mathrm{NbO}_{3}(0.03 \leq \mathrm{x} \leq 0.2)$ samples under visible light irradiation was studied measuring the $\mathrm{H}_{2}$ produced from water splitting with oxalic acid $0.025 \mathrm{M}$ as a sacrificial reagent. As can be seen from the $\mathrm{H}_{2}$ production rate of 35 the different samples (Fig. 5), the photocatalytic activity changes with $\mathrm{Sr}$ content. In particular it seems to increase for $\mathrm{x}=0.2 \rightarrow \mathrm{x}=$ 0.1 , it reaches a maximum for $\mathrm{x}=0.06-0.08$ and it drops again for $\mathrm{x}=0.03$. On average the production rate is $5.95 \mu \mathrm{mol} \mathrm{h} \mathrm{h}^{-1}$ and the maximum rate for $\mathrm{x}=0.06$ is $8.21 \mu \mathrm{mol} \mathrm{h} \mathrm{h}^{-1}$. Time 40 course of $\mathrm{H}_{2}$ evolution diagrams for the samples $\mathrm{x}=0.2 \& \mathrm{x}=0.1$ are shown in Fig. 7. In the same diagram the stable nature of the material can be observed. The steady performance, test after test, gives evidence of no changes taking place on the morphology and surface of the sample. The material stability was proved by XRD 45 and XPS analysis before and after testing. The same behaviour in photocatalytic activity as seen by their $\mathrm{H}_{2}$ production rate is exhibited by the ball milled samples too (Fig. 5). 


\section{Cite this: DOI: $10.1039 / \mathrm{c0xx00000x}$}

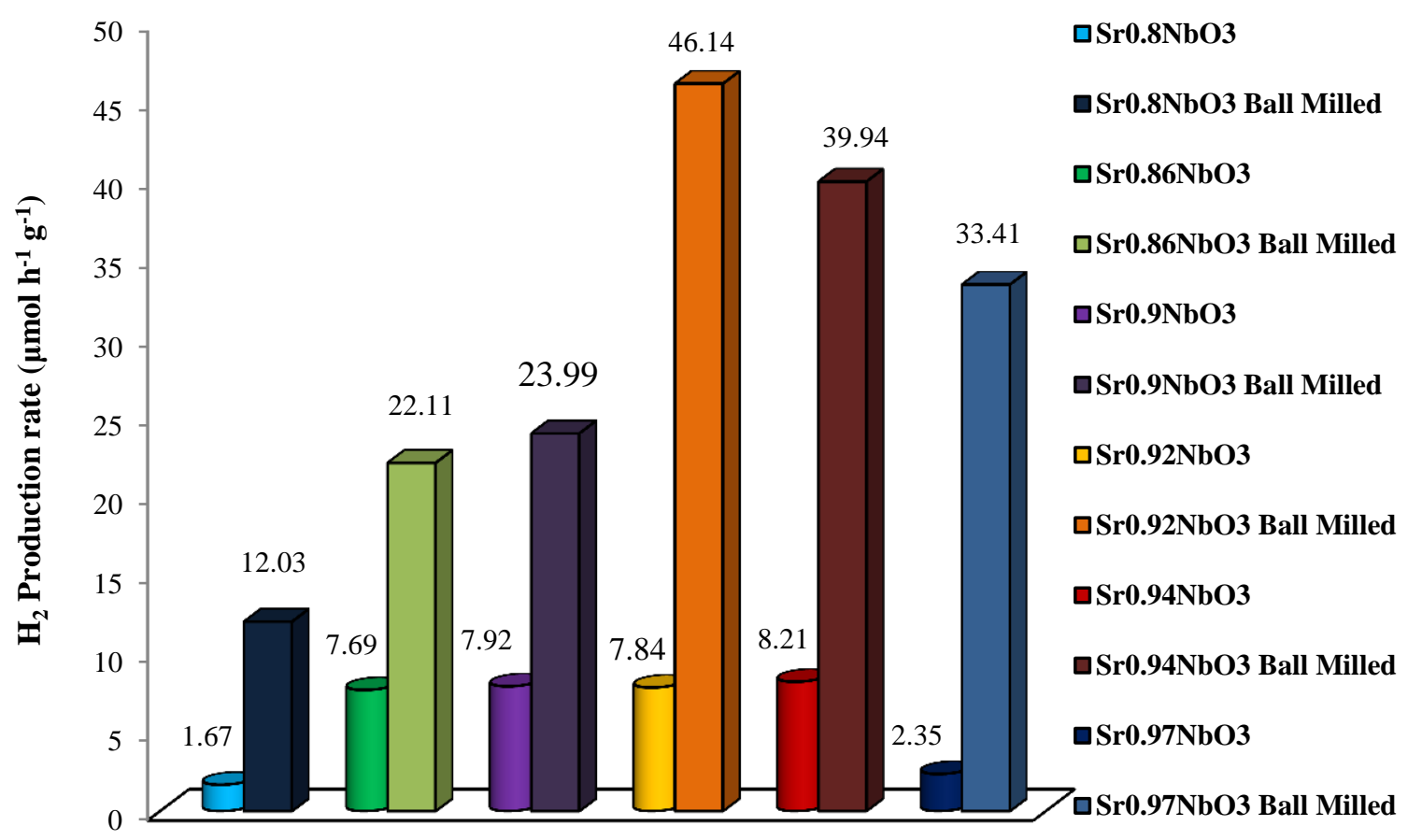

Fig. 5 Comparison of the photocatalytic $\mathrm{H}_{2}$ production rate $\left(\mu \mathrm{mol} \mathrm{h} \mathrm{h}^{-1} \mathrm{~g}^{-1}\right)$ from water splitting under visible light irradiation in aqueous oxalic acid solution $(0.025 \mathrm{M})$ between $\mathrm{Sr}_{1-\mathrm{x}} \mathrm{NbO}_{3}$ samples before and after ball milling. Columns represent the initial sample and boxes represents the same sample after ball milling.

5 Again the activity increases until $x=0.06-0.08$ where a maximum is observed and then drops for $x=0.03$. Additionally, a significant increase in the samples' performance can be seen after ball milling as opposed to the performance of the same samples before ball milling. Specifically, the performance increased from 10 a minimum of 3 times (for $x=0.14$ ) to a maximum of 14 times (for $\mathrm{x}=0.03$ ). The average production rate for the ball milled samples is $23.60 \mu \mathrm{mol} \mathrm{h}^{-1} \mathrm{~g}^{-1}$ (5 times higher than the average rate for non ball milled samples) and the maximum is $46.14 \mu \mathrm{mol} \mathrm{h}$ $\mathrm{g}^{-1}$ given by $\mathrm{x}=0.08$. It is clear enough that both the $\mathrm{Sr}$ content of
15 the sample, which influences the structure of the material, as well as the surface area of the sample play an important role in determining the photocatalytic performance that it exhibits. Moreover, as shown below, the $\mathrm{Sr}$ content at the material's surface may also be important, since perovskites tend to create a ${ }_{20} \mathrm{Sr}$ rich layer on their surface ${ }^{40,41}$. All these parameters influence greatly the photocatalytic activity and it is important to study each of them separately and to determine the relative importance of structure, surface area and surface nature. 


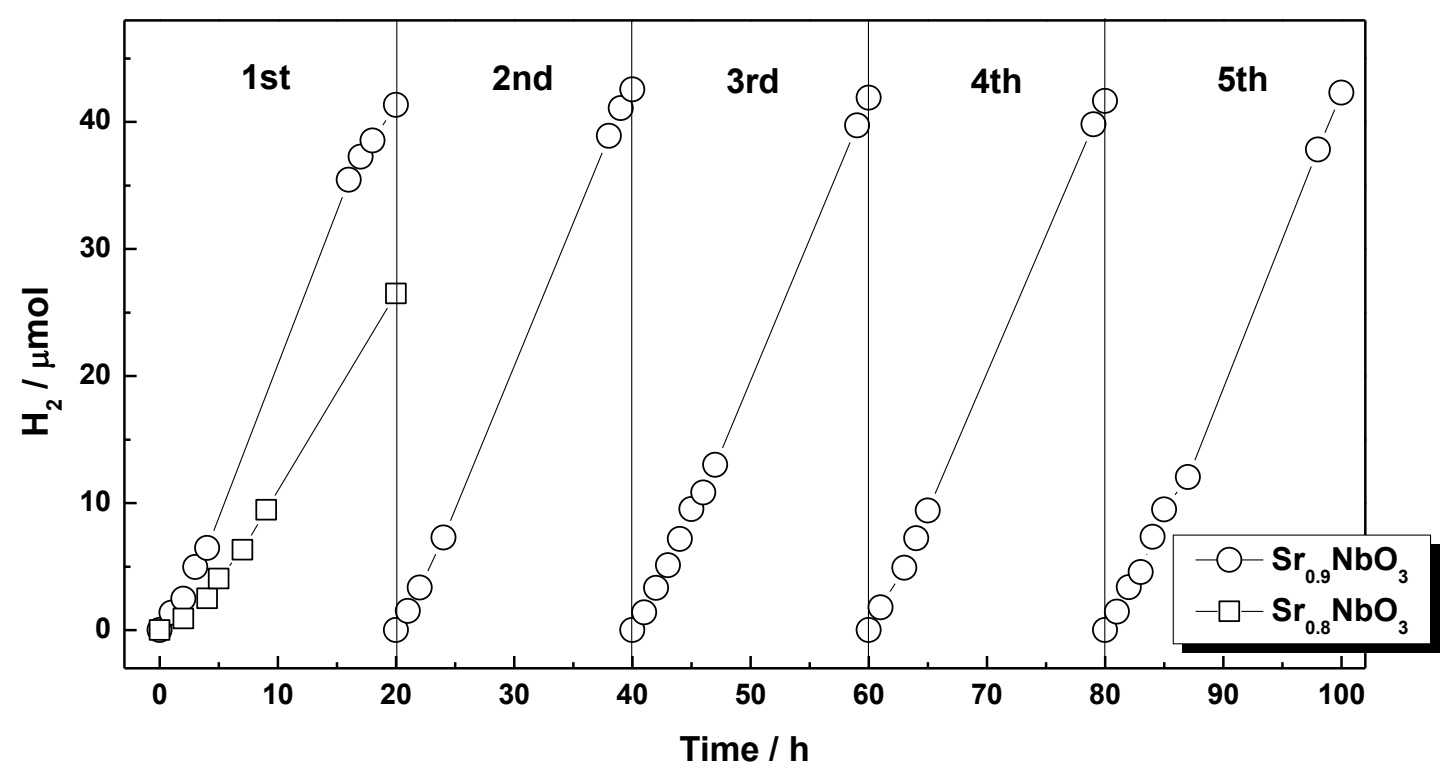

Fig. 6 Typical time courses of $\mathrm{H}_{2}$ evolution in aqueous oxalic acid solution $(0.025 \mathrm{M})$ with $0.2 \mathrm{~g}$ catalyst, reactor was purged with Ar every 20 hours to repeat the experiment. Results for $(1-\mathrm{x}=0.9)$ have been presented in our previous work ${ }^{1}$.

\section{The effect of Sr content to the photocatalytic performance}

Increasing Sr content in the structure not only increases unit cell parameters, which leads to less orbital overlapping, but also triggers structure changes from cubic to orthorhombic when exceeding $(1-\mathrm{x})=0.92$. In the distorted, orthorhombic phase of 10 strontium niobate, $\mathrm{d}$ orbital overlap is reduced in comparison with cubic symmetric phase. As a result, the charge mobility decreases with increasing Sr content. Such a fact is a downside for the photocatalytic activity of a material that has a strong metallic behaviour, since the prevention of electrons and holes 15 recombination is thought to be related to the high charge mobility. On the other hand, increasing the $\mathrm{Sr}$ content of the material leads to more reduced $\mathrm{Nb}$ cations, so that the bands are enriched with electrons occupying higher energy states. In addition, shifting of the UV-VIS absorbance peaks of the material 20 towards higher frequency (blue shift) was observed when the $\mathrm{Sr}$ content in the samples increased, suggesting increased energy gaps (Eg) (Fig. 7). This implies that electrons in the higher $\mathrm{Sr}$ content samples are likely to be excited to higher energy levels with higher reducing power. Such effect is an upside for the 25 photocatalytic activity, particularly for photoreduction. According to experimental data, in the range of $\mathrm{Sr}$ content $(1-\mathrm{x})=$ 0.8 to 0.92 where cubic structure is still maintained, increasing $\mathrm{Sr}$ content has a positive effect to the photocatalytic activity. While further increase of the $\mathrm{Sr}$ content seems to have a negative effect 30 to the photocatalytic activity as the crystal structure changes from cubic to orthorhombic and becomes more distorted.

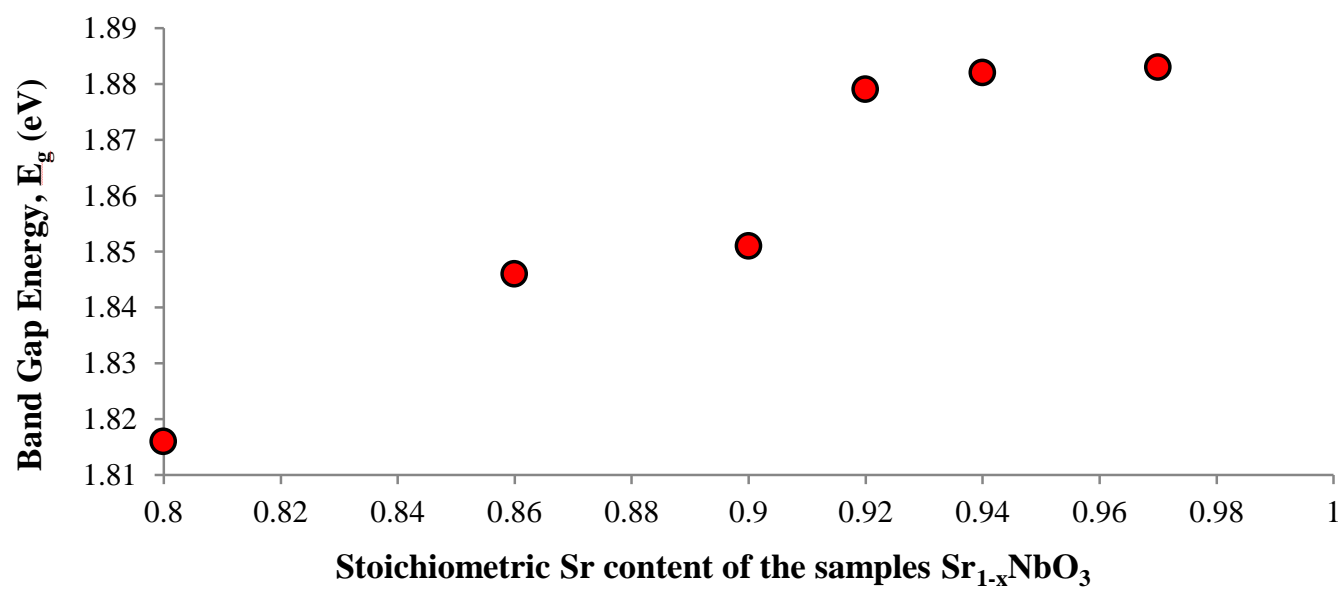


Fig. 7 Band gap energy as calculated by Kubelka - Munk transformations from the UV-VIS spectras of the samples plotted against the stoichiometric Sr content of every $\mathrm{Sr}_{1-\mathrm{x}} \mathrm{NbO}_{3}$ sample.
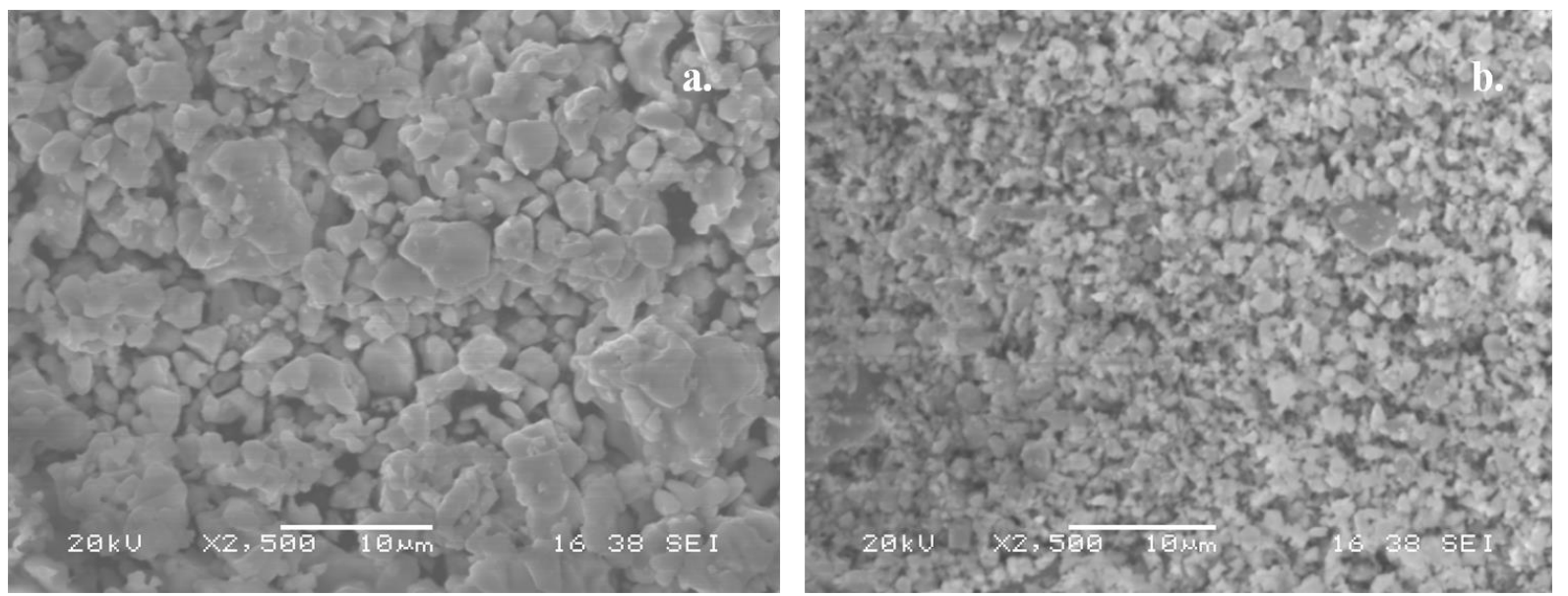

Fig. 8 SEM images of the sample $\mathrm{Sr}_{0.9} \mathrm{NbO}_{3}$ before (a.) and after the ball milling process (b.)

\section{The effect of surface area on photocatalytic activity}

The surface area increases greatly after the ball milling of the samples. The XRD patterns of the samples remain the same revealing that the material suffers no chemical transformation, however the peaks are broadened due to the particle size 10 decrease. The unchanged quality of the sample, as well as the achieved particle size decrease is observed in the SEM pictures too (Fig. 8). The particle size distribution before and after ball milling, after two minutes of sonication in order for the agglomeration to break down and particle size distribution to be 15 more obvious, can be seen in Fig. 9. Before ball milling most of the particles have particle size of $\sim 6 \mu \mathrm{m}$ and after the ball milling process the size drops to a few hundred nm for the majority of the particles. From the broadening of the XRD peaks after ball milling and by using the Debye Scherrer equation the particle size 20 for $(1-\mathrm{x})=0.92$ was found $72 \mathrm{~nm}$ and for $(1-\mathrm{x})=0.94$ was found $92 \mathrm{~nm}$. The performance of the samples greatly increases after ball milling. However, the plot of the $\mathrm{H}_{2}$ production rate of the samples against their surface area in $\mathrm{m}^{2} / \mathrm{g}$ (Fig. 10), as measured by BET analysis, reveals that there is no simple dependence of 25 the performance upon the surface area and other factors, such as the $\mathrm{Sr}$ content are also important.

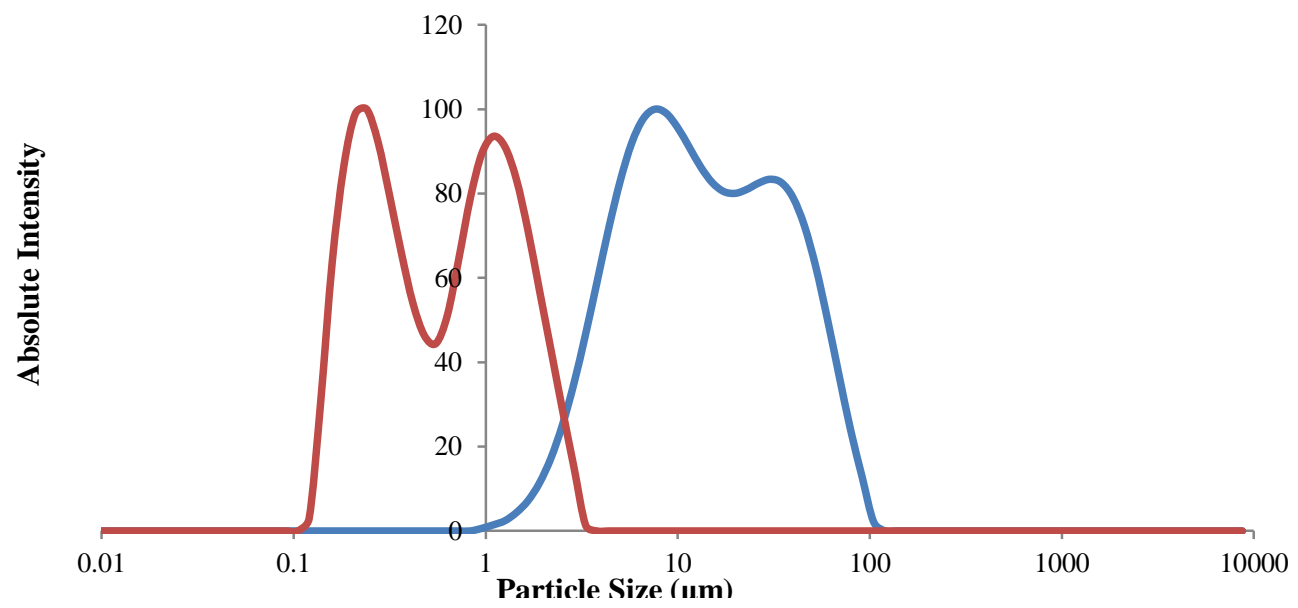

Fig. 9 Particle size distribution before (blue line) and after (red line) ball milling, done in views of decreasing the material's particle size and increase the surface area. Solutions sonicated for two minutes to break down agglomeration. 
Cite this: DOI: $10.1039 / \mathrm{c} 0 \mathrm{xx} 00000 \mathrm{x}$
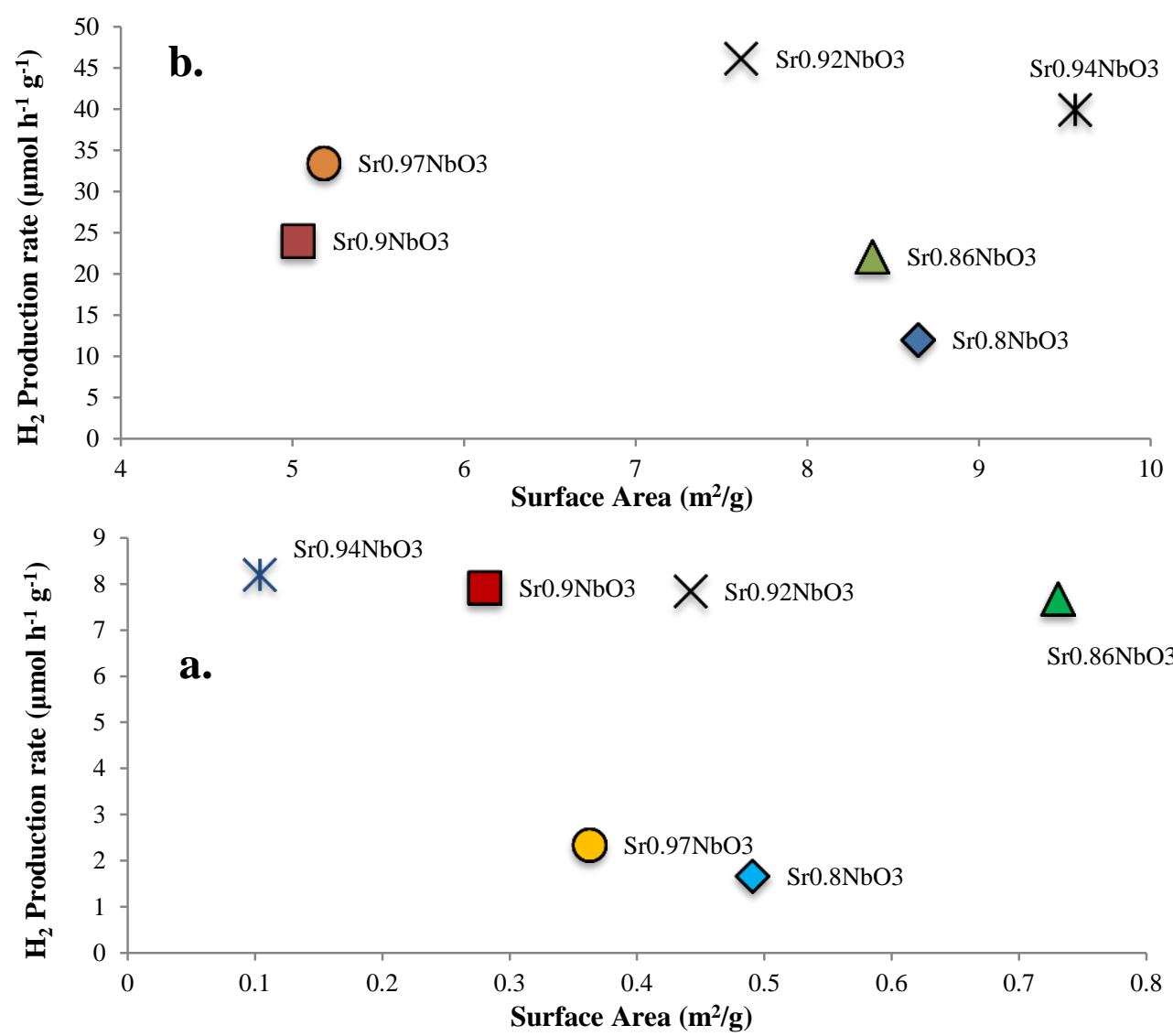

Fig. 10 Photocatalytic performance of the samples in $\mathrm{H}_{2}$ production rate plotted against the surface area of a. un-ball milled and b. ball milled samples. 
Cite this: DOI: $10.1039 / \mathrm{c0xx00000x}$

www.rsc.org/xXXXXX

ARTICLE TYPE
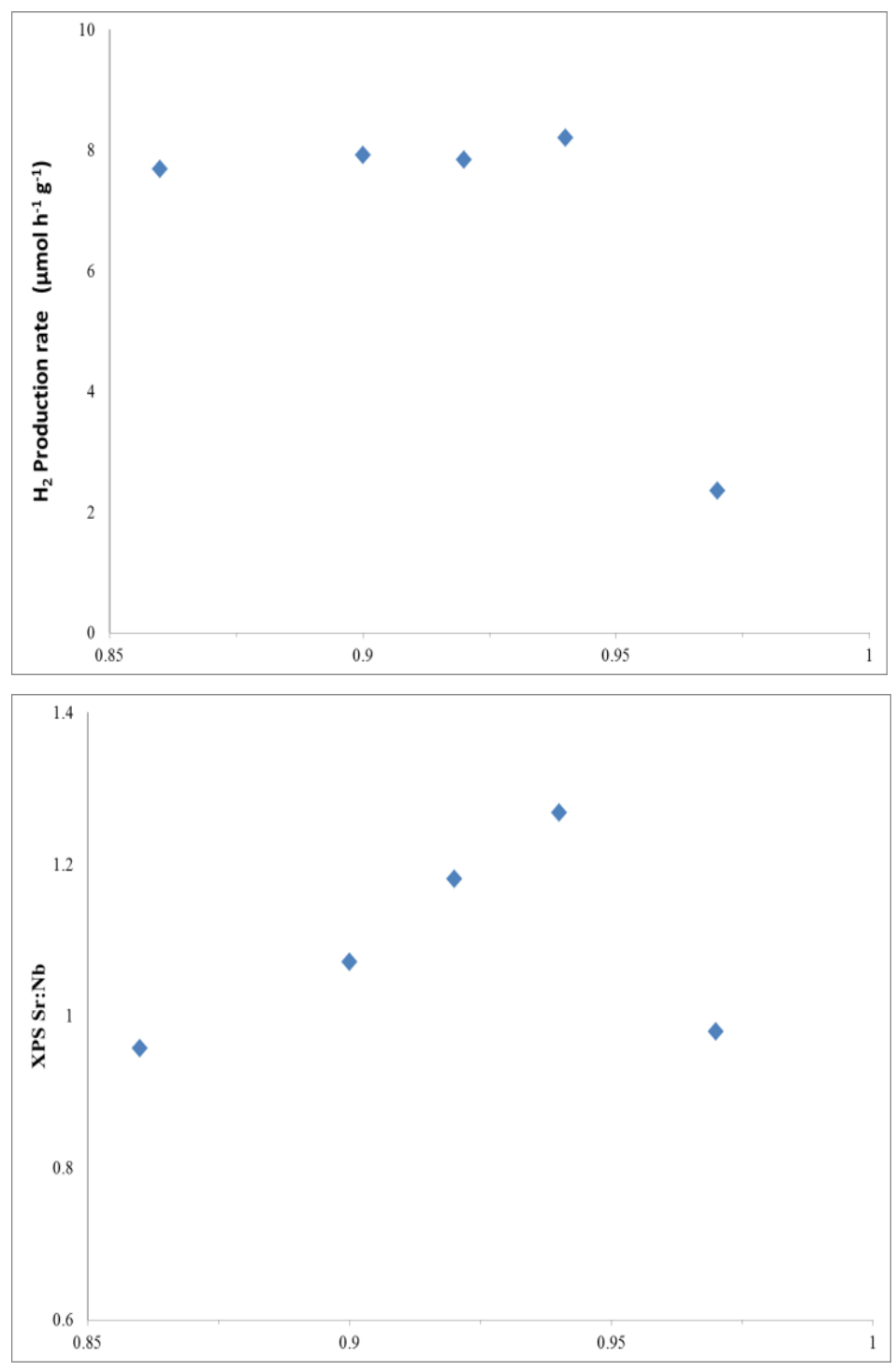

Fig. 11 (a) The photocatalytic $\mathrm{H}_{2}$ production rate of the samples, (b) oxygen stoichiometry as determined from TGA in air, similar to Fig. 2 (c)The $\mathrm{Sr}: \mathrm{Nb}$ ratio content found on the surface of the samples by XPS, 5 all plotted against $\mathrm{Sr}$ content $(1-\mathrm{x}=0.86,0.9,0.92,0.94,0.97)$.

\section{The influence of chemistry on the photocatalytic activity}

The relationships between hydrogen production rate, oxygen content and surface chemistry are examined in Fig. 11. XPS measurements clearly show $\mathrm{Sr}$ surface enrichment for all the 10 samples. Such $\mathrm{Sr}$ enrichment has previously been reported in a number of different perovskite systems ${ }^{40,41}$. The $\mathrm{Sr}$ content of the surface is found to vary according to the bulk stoichiometric $\mathrm{Sr}$ content of the sample up to the point $(1-\mathrm{x})=0.94$. There is clear correlation with hydrogen production rate; however, changes in

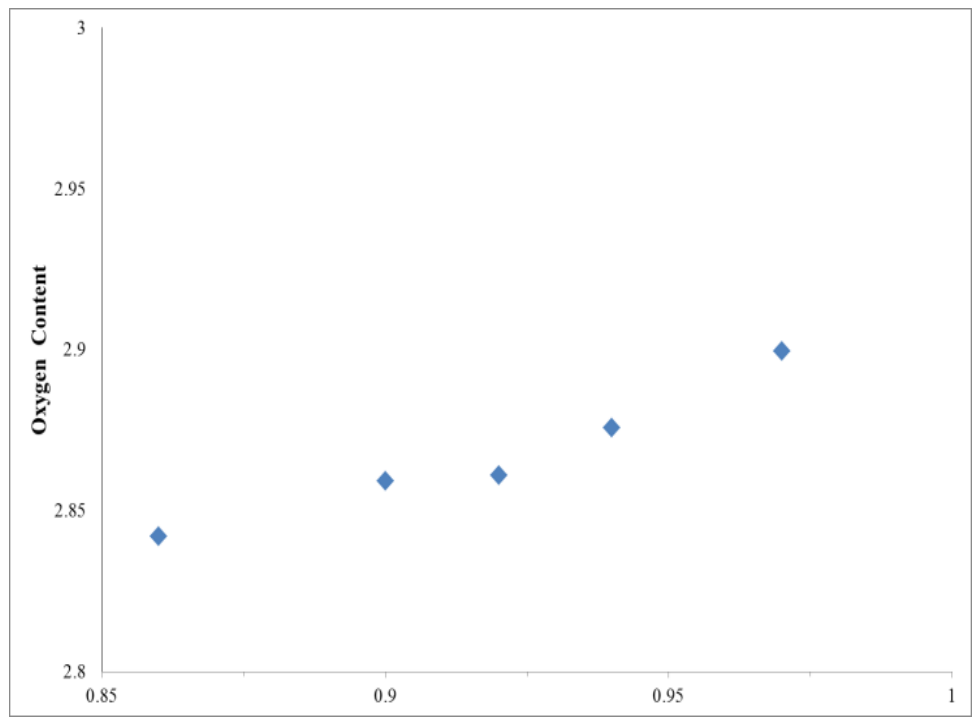

15 both $\mathrm{Sr}$ enrichment and hydrogen production rate are thought to depend upon unit cell symmetry suggesting the correlation is indirect. The actual bulk oxygen content of the samples $\left(\mathrm{Sr}_{1-}\right.$ ${ }_{x} \mathrm{NbO}_{3-\delta}$ ) was calculated from the weight gained during TGA analysis, after heating up to $900{ }^{\circ} \mathrm{C}$ under air and then cooling 20 back to room temperature. Oxygen deficiency decreases with deceasing A-site deficiency but seems not related to photocatalytic activity.

\section{Conclusions}

${ }_{25}$ Strontium niobate is a metallic A-site deficient perovskite oxide that strongly absorbs light of $1.9 \mathrm{eV}$ energy and above. It is photocatalytically active for hydrogen evolution without a precious metal co-catalyst, although a sacrificial element is generally required. Activity depends on several factors and 30 increases with particle surface area and average $\mathrm{Nb}$ oxidation state, which increases as $\mathrm{Sr}$ deficiency decreases. These tendencies are disrupted when the structure distorts from cubic to orthorhombic at high $\mathrm{Sr}$ contents where activity decreases. The materials is stable against photocatalytic degradation and reaction 35 with water at ambient temperatures, which might not be expected for such a low $\mathrm{Nb}$ oxidation state material. Absorption of light by interband transitions in a strongly coloured metallic oxide is not surprising; however, that this light can yield excitations able to drive photocatalytic reactions is surprising as recombination 40 would be expected to dominate. In a semiconductor a depletion layer near the surface allows photocarrier separation, but this would not be possible in a metallic oxide. The observation of $\mathrm{Sr}$ enrichment at the surface via XPS does suggest an answer to these problems as the Sr-rich layer would serve to chemically ${ }_{45}$ protect the underlying reduced $\mathrm{Nb}$ oxide and would provide a surface potential barrier to facilitate charge separation. 


\section{Acknowledgements}

We are very pleased to have this article linked to the special issue in honour of David Cole-Hamilton, an excellent colleague of nearly 20 years. We thank EPSRC and NSF for financial support. PE thanks Sasol ${ }_{5}$ UK and ETP for studentship support. XX and JTSI also thank Anglo Platinum for research support.

\section{References}

1 X. Xu, C. Randorn, P. Efstathiou and J. T. S. Irvine, Nat. Mater., 2012, 11, 595.

10

2 K. Maeda, K. Teramura, N. Saito, Y. Inoue, H. Kobayashi and K. Domen, Pure Appl. Chem, 2006, 78, 2267.

3 K. Maeda and K. Domen, J. Phys. Chem. C, 2007, 111, 7851.

4 Y. Sasaki, A. Iwase, H. Kato and A. Kudo, J. Catal., 2008, 85 259, 133.

155 A. Kudo, MRS Bull., 2011, 36, 32.

6 H. J. Yun, H. Lee, N. D. Kim, D. M. Lee, S. Yu and J. Yi, ACS Nano., 2011, 5, 4084.

7 M. Higashi, R. Abe, A. Ishikawa, T. Takata, B. Ohtani and K. Domen, Chem. Lett., 2007, 37.

208 A. Iwase, Y. H. Ng, Y. Ishiguro, A. Kudo and R. Amal, J. Am. Chem. Soc., 2011, 133, 11054.

9 A. Sclafani and J. M. Herrmann, J. Phys. Chem., 1996, 100, 13655.

10 M. Anpo, Catal. Surv. Japan, 1997, 1, 169.

11 A. Fujishima, T. N. Rao and D. A. Tryk, J. Photochem. Photobiol., C, 2000, 1, 1.

12 A. Fujishima and X. Zhang, C. R. Chim., 2005, 9, 750.

13 I. Sopyan, M. Watanabe, S. Murasawa, K. Hashimoto and A. Fujishima, J. Photochem. Photobiol., A, 1996, 98, 79.

3014 X. Chen, L. Liu, P. Y. Yu and S. S. Mao, Science, 2011, 331, 746.

15 I. M. Arabatzis, T. Stergiopoulos, M. C. Bernard, D. Labou, S. G. Neophytides and P. Falaras, Appl. Catal., B, 2003, 42, 187.

16 E. Paparazzo, Catal. Lett., 2010, 140, 147.

3517 B. Liu, L. Wen and X. Zhao, Sol. Energ. Mat. Sol. C., 2008, 92, 1.

18 A. Kudo, Catal. Surv. Asia, 2003, 7.

19 S. Bassaid, D. Robert and M. Chaib, Appl. Catal., B, 2009, 86, 93.

20 R. Amade, P. Heitjans, S. Indris, M. Finger, A. Haeger and D. Hesse, J. Photochem. Photobiol., A, 2009, 207, 231.

21 S. Ikeda, A. Tanaka, K. Shinohara, M. Hara, J. N. Kondo, K.-i. Maruya and K. Domen, Microporous Mater., 1997, 9, 253.

22 S. Vajpai, R. Dube and M. Sharma, Journal of Materials Science, 2009, 44, 4334.

23 J. R. Darwent and A. Mills, J. Chem. Soc., Faraday Trans. 2, $1982, \mathbf{7 8}, 359$

24 S. Tokunaga, H. Kato and A. Kudo, Chem. Mater., 2001, 13, 4624.

25 R. Konta, T. Ishii, H. Kato and A. Kudo, J. Phys. Chem. B, 2004, 108, 8992.

26 N. Peng, J. T. Irvine and A. G. Fitzgeral, J. Mater. Chem., 1998, 8, 1033.

27 K. Isawa, J. Sugiyama, K. Matsuura, A. Nozaki and H. Yamauchi, Phys. Rev. B, 1993, 47, 2849.

55

28 B. Hessen, S. A. Sunshine, T. Siegrist and R. Jimenez, Mater. Res. Bull., 1991, 26, 85.

29 D. Ridgley and R. Ward, J. Am. Chem. Soc., 1955, 77, 6132.

30 P. Kubelka and F. Z. Munk, Tech. Phys., 1931, 12, 593.

6031 R. B. Macquart, B. J. Kennedy and M. Avdeev, Journal of Solid State Chemistry, 2010, 183, 2400.

32 H. Hannerz, G. Svensson, S. Y. Istomin and O. G. D'Yachenko, J. Solid State Chem., 1999, 147, 421.

33 N. Peng and J. T. S. Irvine, Phys. C Supercond., 1997, 282.

6534 O. G. D'Yachenko, S. Y. Istomin, A. M. Abakumov and E. V. Antipov, Inorg. Mater., 2000, 36, 247.

35 E. Konysheva and J. T. S. Irvine, J. Mater. Chem., 2008, 18, 5147.
36 R. D. Shannon and C. T. Prewitt, Acta. Crystall. B-Stru., 1970, B 26, 1046.

37 D. R. Lide and H. P. R. Frederikse, Handbook of Chemistry and Physics, 76 edn., The Chemical Rubber Publishing Company, 1995-1996.

38 R. A. Serway, C. Vuille and J. S. Faughn, College Physics, 8 edn., Cengage learning, Inc., 2009.

39 H. O. Finklea, J. Chem. Educ., 1983, 60, 325.

40 E. Mutoro, E. J. Crumlin, H. Pöpke, B. Luerssen, M. Amati, M. K. Abyaneh, M. D. Biegalski, H. M. Christen, L. Gregoratti, J. Janek and Y. Shao-Horn, J. Phys. Chem. Lett., $2011,3,40$.

41 T. T. Fister, D. D. Fong, J. A. Eastman, P. M. Baldo, M. J. Highland, P. H. Fuoss, K. R. Balasubramaniam, J. C. Meador and P. A. Salvador, Appl. Phys. Lett., 2008, 93, 15. 